\title{
"Putback" is the essence of bidirectional programming
}

\author{
FISCHER Sebastian ${ }^{1}$, HU Zhenjiang ${ }^{2,4,5 *} \&$ PACHECO Hugo ${ }^{3}$ \\ ${ }^{1}$ Christian-Albrechts-Universität of Kiel, Germany; \\ ${ }^{2}$ National Institute of Informatics, Japan; \\ ${ }^{3}$ Cornell University, USA; \\ ${ }^{4}$ The Graduate University for Advanced Studies (SOKENDAI), Japan; \\ ${ }^{3}$ Peking University, China \\ Received ; accepted
}

\begin{abstract}
Bidirectional transformations (BXs), programs with a forward transformation and a backward transformation that maintain consistency between input and output, are routinely written in ways that do not let programmers specify their behavior completely. Several bidirectional programming languages exist to aid programmers in writing BXs with increased maintainability but decreased expressiveness.

Such languages allow programmers to write BXs as one program for both directions, which is easier to maintain than separate programs for each direction. However, the maintainability provided by existing bidirectional languages comes at the cost of expressiveness because the ambiguity of synchronization is solved by default strategies which are hidden from programmers. The programmers' inability to influence synchronization strategies has led to the proposal of a vast number of approaches that consider tailor-made synchronization strategies for particular applications.

In this paper, we argue that such ambiguity is essential for BX and advocate that the synchronization strategy should not be hidden from programmers but considered from the start. We propose a novel approach to specifying so-called well-behaved bidirectional programs by their backward transformations, capable of expressing all aspects of a BX while retaining maintainability.

Soundness of our approach results from a systematic analysis, based on existing mathematical concepts, of the instrumental laws of well-behaved BXs. We show that well-behaved BXs are uniquely determined by their backward transformations and corresponding forward transformations can be obtained for free.
\end{abstract}

Keywords Bidirectional Transformation, Software Adaption and Coevolution, Bidirectional Programming

Citation Fischer S, Hu Z, Pacheco H. "Putback" is the Essence of Bidirectional Programming. Sci China Inf Sci, 2014, 57: $\operatorname{xxxxxx} 22$, doi: $\operatorname{xxxxxxxxxxxxxx}$

\section{Introduction}

Bidirectional transformation (BX for short) [6, 16, originated from the view updating mechanism in the database community [1, 17, 11, has been recently attracting a lot of attention from researchers in the communities of programming languages and software engineering since the pioneering work of Foster et

* Corresponding author (email: hu@nii.ac.jp) 
Fischer S, et al. Sci China Inf Sci $\quad$ xxxxxx 2014 Vol. 57 xxxxxx:2

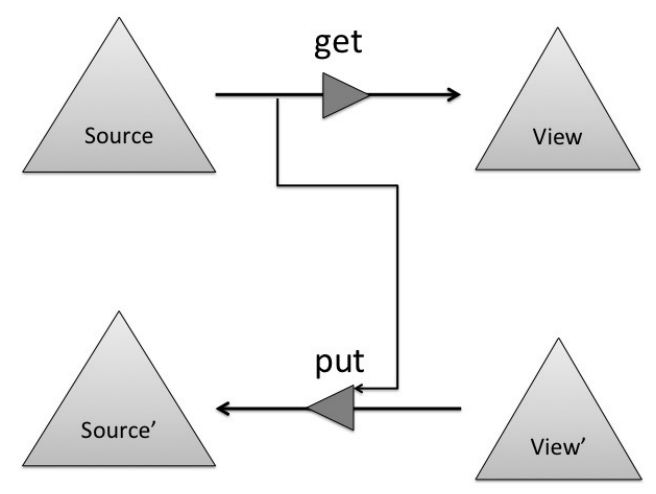

Figure 1 Forward and backward directions in BXs.

al. [10] on a combinatorial language for bidirectional tree transformation. BX provides a novel mechanism for synchronizing and maintaining the consistency of information between input and output and has seen many interesting applications, including the synchronization of replicated data in different formats [10], presentation-oriented structured document development [17, interactive user interface design [25], or coupled software transformation [21].

\subsection{Bidirectional transformation (BX)}

A BX consists of a pair of transformations: the forward transformation is used to produce a target view from a source, while the backward transformation is used to "put back" modifications on the view to the source. To allow the forward transformation to discard information when producing a view, the backward transformation is supplied the original source in addition to the updated view. This situation is depicted in Figure 1 where, as is customary, the forward transformation is called get (also know as view function) and the backward transformation is called put (shorthand for "putback").

Example 1. As a simple example for a BX, consider a forward function getFirst that selects the first component of a pair and a corresponding backward function putFirst that updates the first component and retains the second component from the original pair. They can be defined as follows:

$$
\begin{aligned}
& \text { getFirst }(x, y)=x \\
& \text { putFirst }(x, y) z=(z, y)
\end{aligned}
$$

Not every combination of get and put functions forms a reasonable BX. Their definitions need to fit together such that one constitutes the opposite direction of the other. Put formally, the get and put functions should be well-behaved in the sense that they satisfy the following GETPUT and PUTGET laws:

$$
\begin{aligned}
& \text { put } s(\text { get } s)=s \\
& \text { get }(\text { put s } v)=v
\end{aligned}
$$

GetPut

PutGet

The GETPUT property requires that not changing the view shall be reflected as not changing the source, while the PUTGET property requires all changes in the view to be completely reflected to the source so that the changed view can be computed again by applying the forward transformation to the changed source.

\subsection{Bidirectional programming}

Bidirectional programming is to develop well-behaved BXs to solve various synchronization problems. A straightforward approach to bidirectional programming is to write two unidirectional transformations. Although this ad hoc solution provides full control over both forward and backward transformations and 
can be realized using standard programming languages, it scales badly for non-trivial transformations and easily becomes expensive and error-prone: not only do we have to write two transformations instead of a single one, but the two transformations must be shown to satisfy the well-behavedness laws. Also, it is a maintenance problem if a modification to one of the transformations requires a redefinition of the other transformation as well as a new proof of the laws.

To ease bidirectional programming and to enable maintainable bidirectional programming, it is preferable to write just a single program that can denote both transformations, which has motivated the following two methods:

- Bidirectionalization of forward transformation. This is to allow users to write the forward transformation in a familiar (unidirectional) programming language and derive a suitable backward transformation through bidirectionalization techniques [19, 22, 32, 24, 31, 13.

- Design of domain-specific BX languages. This is to instruct users to write a program in a particular bidirectional programming language [10, 4, 3, 27, 14, 15, 28], from which both transformations can be derived.

What both methods have in common is that one writes a forward transformation in a unidirectional language or a domain-specific BX language, and a corresponding good backward transformation can be automatically derived, which makes bidirectional programming easy and maintainable. Despite these advantages, there is an impractical assumption that

for a forward transformation get, it is sufficient to derive a "suitable" put that can be combined to form a well-behaved bidirectional transformation.

In general a get function may not be injective, so there may exist many possible put functions that can be combined with get to form a BX. Also, the most "suitable" or "best" backward transformation may be hard to find and to justify. There is no clear consensus on the best requirements even for well-studied domains [5].

To understand the inherent ambiguity in possible "putback" functions, consider, as an example, the following forward transformation ${ }^{1)}$

$$
\text { getEvens } n s=\text { filter even } n s
$$

It returns those elements of a list of numbers which are even.

$$
\text { getEvens }[2,3,5,6]=[2,6]
$$

What is a corresponding put? If 6 is eliminated from the result, leaving the view list [2], there are many well-behaved ways of putting back this elimination to the source. For example, we could delete the number 6 from the source $[2,3,5,6]$ to obtain the updated source $[2,3,5]$ or change 6 to $n$ obtaining $[2,3,5, n]$, where $n$ is an arbitrary odd number. Keller [19] argues that many of these "putback" functions are incomparable, and that there is no reasonable approach to say which one is the best for all situations.

It is this unavoidable ambiguity of put that makes bidirectional programming difficult to be used in practice due to possibly unpredictable behaviors, and that has led to the boom of current bidirectional frameworks, in an attempt to answer the needs of particular bidirectional applications [6, 16].

\subsection{Putback-based bidirectional programming}

So far, bidirectional programming has been focused on writing the forward transformation (or a bidirectional program that resembles the forward transformation), and then trying to derive a suitable (but less obvious) backward transformation that embodies a specific way to solve the ambiguity of update translation (i.e., an update strategy) [19, 2, 28.

In this paper, we argue that the update strategy should be considered from the start, and propose a novel putback-based approach to bidirectional programming: writing put and deriving get (i.e., specifying

1) We use Haskell syntax for our examples as we discuss further in Section 3.1 The filter function is predefined and selects all elements from a list that satisfy the given predicate - in this case even, which is also predefined. 
the intended backward transformation that best suits particular purposes, and deriving the forward transformation). The new approach attains the advantages of writing a single program to specify a BX, offering better maintenance. It also enjoys an important feature that, in sharp contrast to bidirectional programming based on get where get is not sufficient to determine put, bidirectional programming based on put has the potential to describe all intentions of BXs, since there is only one get that can be combined with a given put to form a well-behaved BX.

There are two major difficulties in constructing a framework for putback-based bidirectional programming. One is to clarify sufficient and necessary conditions on the put function such that it can be used to describe all intentions of a well-behaved BX while guaranteeing existence and uniqueness of the corresponding get function. The other difficulty is how to automatically derive the unique get from put in practice. Our main technical contributions can be summarized as follows.

- We clarify the necessary conditions on putback functions of well-behaved BXs in Section 3 and use these conditions to characterize different classes of BXs in Section 4.

- We formally prove in Section 4 that for a given put function that satisfies the identified necessary conditions, the corresponding get function is unique in each considered class of BXs. Hence, the get function is redundant and can be derived automatically, retaining maintainability and offering full control.

- We describe in Section 5 a prototype implementation for deriving get from put in the functional logic programming language Curry [12] and demonstrate how to program putback style programs that implement different update strategies for transformations resembling database queries.

In the rest of the paper, we begin in Section 2 by discussing the considered classes of BXs more formally and provide corresponding intuitions using examples that we also use to show the ambiguity of backward transformations for given forward transformations. We discuss related work in Section 6 and provide our conclusions together with possibilities for future work in Section 7.

\section{Classes of BXs}

In this section, we review different classes of BXs, discussing their laws both equationally and intuitively. Example transformations in this section are minimalistic to highlight the essential differences between different classes of BXs.

\subsection{Well-behaved BXs}

Foster et al. 10, call BXs that satisfy the GETPUT and PUTGET laws well-behaved.

Definition 1 GETPUT law). The GETPUT law, formulated in Section 1.1, describes a property of calling the forward function get before the backward function put. It is depicted in Figure 2.

The put function should yield an unmodified source when passing the view obtained by get unchanged along with the original source. This property captures the intuition that if no view update takes place the source should not be updated either.

Definition 2 PUTGeT law). The PUTGET law, formulated in Section 1.1, describes a property of calling the backward function put before the forward function get. It is depicted in Figure 3.

When passing a source obtained by put to get, then get should yield the same view that was passed to put. This property captures the intuition that view updates are reflected to the source by put and can be observed by get.

The BX defined in Example1 is well-behaved because it satisfies the GeTPUT and PUTGET laws. To verify the GETPUT law, consider the following equations:

$$
\begin{aligned}
& \text { putFirst }(x, y)(\text { getFirst }(x, y)) \\
= & \{\text { definition of getFirst }\} \\
& \text { putFirst }(x, y) x \\
= & \{\text { definition of } \text { putFirst }\} \\
& (x, y)
\end{aligned}
$$




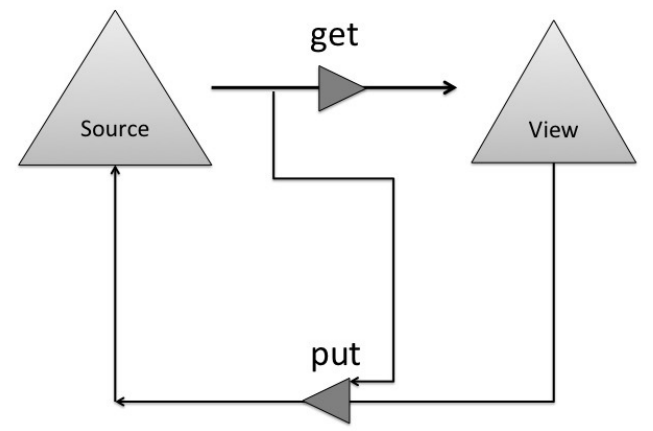

Figure 2 The GetPut law.

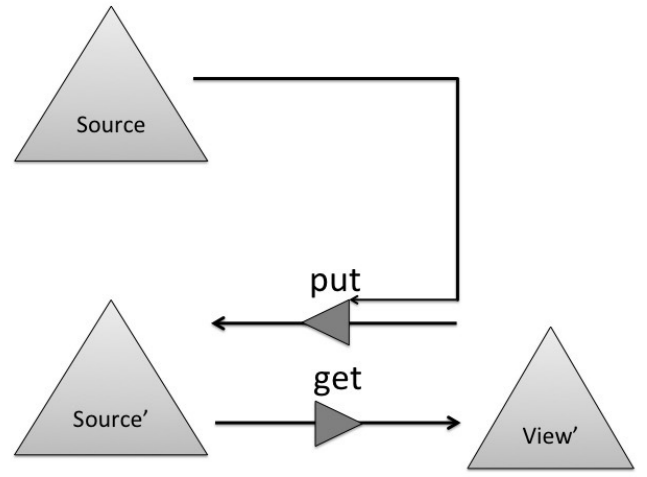

Figure 3 The PutGeT law.

So, indeed, when we update the first component of a pair with its own first component, the pair does not change. To verify the PUTGET law, consider the equations below.

$$
\begin{aligned}
& \text { getFirst }(\text { putFirst }(x, y) z) \\
= & \{\text { definition of putFirst }\} \\
& \text { getFirst }(z, y) \\
= & \{\text { definition of getFirst }\}
\end{aligned}
$$

So, indeed, if we query the first component of a pair with an updated first component, we get back the value used for updating.

Example 2 (Change counter). As another example of a well-behaved BX, consider the combination of getFirst with the following backward function:

$$
\text { putFirstCount }(n, c) m=\text { if } n==m \text { then }(m, c) \text { else }(m, c+1)
$$

This function increments the second component of a pair whenever we change its first component. It is easy to verify both GETPUT and PUTGET laws.

\subsection{Very well-behaved BXs}

Foster et al. [10] call well-behaved BXs that also satisfy the PUTPUT law very well-behaved.

Definition 3 (PutPut law). The PUTPUT law describes a property of calling the backward function put twice with different views.

$$
\text { put }\left(\text { put } s v^{\prime}\right) v=\text { put } s v
$$

PutPut

When put is called twice in a row, then the result should be the same as the result of the second call with the updated source replaced by the original. This property captures the intuition that view updates are independent of each other.

The BX defined in Example 1 is very well-behaved, as can be verified by the following equations:

$$
\begin{aligned}
& \text { putFirst }\left(\text { putFirst }(x, y) z_{1}\right) z_{2} \\
= & \{\text { definition of putFirst }\} \\
& \text { putFirst }\left(z_{1}, y\right) z_{2} \\
= & \{\text { definition of } \text { putFirst }\} \\
& \left(z_{2}, y\right) \\
= & \{\text { definition of } \text { putFirst }\} \\
& \text { putFirst }(x, y) z_{2}
\end{aligned}
$$


The first update (using the value $z_{1}$ ) does not influence the second update (using $z_{2}$ ) because updates completely overwrite the effect of previous updates.

The BX defined in Example 2 is not very well-behaved because the PUTPUT law is violated if two updates in a row change the first component of the pair more often than the second update alone.

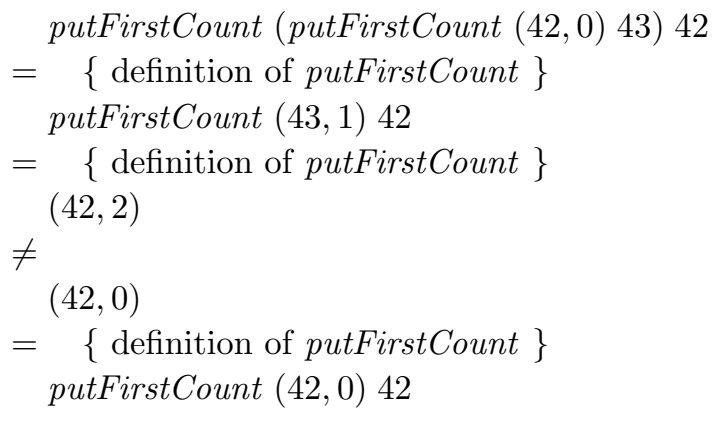

Different updates are not independent because their effect on the counter cannot be overwritten.

Example 3 (Maintaining difference). As a second example of a BX that is very well-behaved, consider the function getFirst together with the following backward function that maintains the original difference of the components of a pair when updating the first component.

$$
\text { putFirstDiff }(x, y) z=(z, z+y-x)
$$

When updating the first component, the second is also changed such that the difference between the components remains the same. It can be proved that the GETPUT PUTGET, and PUTPUT laws all hold.

Example 3 follows a general pattern to construct very well-behaved BXs by maintaining a constant complement [1] of the view in invocations of the put function. In fact, Foster et al. [10] prove that view updating under constant complement captures every very well-behaved BX.

\subsection{Bijective BXs}

Foster et al. 10. call BXs that satisfy the STRONGGetPut law in addition to the PUTGET law bijective. Definition 4 (StrongGetPut law). The StrongGetPut law is a stronger version of the GetPut law for well-behaved BXs.

$$
\text { put } s^{\prime}(\text { get } s)=s
$$

StrongGetPut

When put is called on the result of get, it should yield the same source that was given to get initially, regardless of what source is used for the update. Together with the PUTGET law, the STRONGGETPUT law captures the intuition that there is a one-to-one correspondence between sources and views implemented by the get and put functions.

The BX defined in Example 1 is not bijective because there is no one-to-one correspondence between pairs and their first components. For example, the following inequality violates STRONGGETPUT;

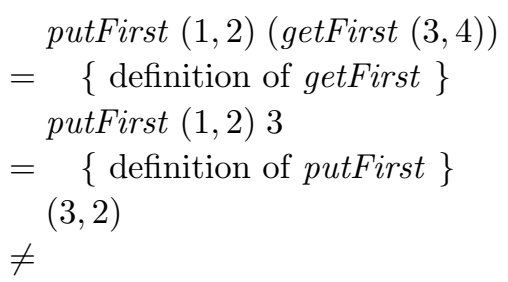

Unlike (very) well-behaved BXs, bijective BXs do not allow to discard information when computing a view from a source. Examples 2 and 3 also do not define bijective BXs because they use the same forward function as Example 1 which discards information. 
Fischer S, et al. Sci China Inf Sci $\quad$ xxxxxx 2014 Vol. 57 xxxxxx:7

\section{Preliminary observations on putback functions}

We now introduce notation and review mathematical concepts that play a role when we characterize the different classes of BXs in Section 4. While doing so, we observe necessary conditions on put functions implied by laws for BXs.

\subsection{Currying, point-free style, and infix operator sections}

We denote function application by juxtaposition (as in the functional programming language Haskell [23]). For example, the application of a function get to a source argument $s$ is written as get $s$. We write functions with multiple arguments in so-called curried style, that is, instead of taking (a tuple of) multiple arguments directly, multi-argument functions take one argument and yield a function for the remaining arguments. As is conventional, function application associates to the left and, hence, the application of a function put to a source argument $s$ and a view argument $v$ is written as follows.

$$
\text { put } s v=(\text { put } s) v
$$

The higher order functions curry and uncurry translate between the curried and uncurried versions of a function.

$$
\begin{aligned}
& \text { curry } f x y=f(x, y) \\
& \text { uncurry } f(x, y)=f x y
\end{aligned}
$$

For example, we can obtain the uncurried version of a put function by calling uncurry and then pass the arguments as a pair.

$$
\text { uncurry put }(s, v)=\text { put } s v
$$

Sometimes, we give type annotations for functions, again in Haskell notation. If sources have type $S$ and views are of type $V$, then curried and uncurried put functions have the following types, respectively:

$$
\begin{aligned}
& \text { put } \quad:: S \rightarrow(V \rightarrow S) \\
& \text { uncurry put }::(S, V) \rightarrow S
\end{aligned}
$$

As is conventional, the function-type constructor associates to the right, so the type $S \rightarrow(V \rightarrow S)$ can be written $S \rightarrow V \rightarrow S$, for short.

We write $i d$ for the identity function on arbitrary types. To highlight a specific argument (and result) type of $i d$, we sometimes write it as a subscript, such as $i d_{S}$ for the identity function on a type $S$.

We write a dot to denote function composition. For example, the composition of a partially applied put function with a get function, applying get to the result of put $s$, is written as follows:

$$
\text { get } \cdot \text { put } s=\lambda v \rightarrow \text { get }(\text { put } s v)
$$

Lambda abstractions, like the one above, define anonymous functions.

Binary functions, such as put, can be enclosed in backquotes to use them as infix operators. Furthermore, partial applications of infix operators can be formed for the first and second argument using parenthesized infix operator sections where only the left (i.e., first) or right (i.e., second) argument is provided. The following examples demonstrate the use of such notation:

$$
\begin{aligned}
& s^{\prime} p u t^{6} v=\text { put } s v \\
& \left(s^{6} p u t^{6}\right)=\lambda v \rightarrow \text { put } s v=\text { put } s \\
& \left({ }^{6} p u t^{6} v\right)=\lambda s \rightarrow \text { put } s v
\end{aligned}
$$

Proposition 1 (Point-free laws for BXs). We can now rephrase some of the laws for BXs reviewed in Section 2 in the so-called point-free style, that is, without mentioning some arguments of functions. 
1. The PutGet law (cf. Definition 2) states the following equation for all sources $s$ :

$$
\text { get } \cdot \text { put } s=i d
$$

2. The PutPut law (cf. Definition 3) states the following equation for all views $v$ and $v^{\prime}$ :

$$
\left({ }^{\prime} p u t^{6} v\right) \cdot\left({ }^{\prime} p u t^{6} v^{\prime}\right)=\left({ }^{\prime} p u t^{6} v\right)
$$

3. The StrongGetPut law (cf. Definition 4) states the following equation for all sources $s$ :

$$
\text { put } s \cdot \text { get }=i d
$$

Reformulating laws in the point-free style will help identify necessary conditions on putback functions using standard mathematical terminology.

\subsection{Mathematical propositions}

This subsection recalls basic mathematical concepts used subsequently and relates them to the previously introduced notation.

Definition 5 (Injectivity and left inverse). A function $f:: A \rightarrow B$ is injective if and only if there is a function $g:: B \rightarrow A$ such that $g \cdot f=i d_{A}$. In this case, $g$ is called left inverse of $f$.

Intuitively, an injective function maps different arguments to different results. As a consequence, the result type is "at least as big as" the argument type.

Definition 6 (Surjectivity and right inverse). A function $f:: A \rightarrow B$ is surjective on $B$ if and only if there is a function $g:: B \rightarrow A$ such that $f \cdot g=i d_{B}$. In this case, $g$ is called right inverse of $f$.

Intuitively, a surjective function yields every value in its result type for some argument. As a consequence, the argument type is "at least as big as" the result type.

Proposition 2 (Injectivity and surjectivity in BXs). Certain laws for BXs impose injectivity and surjectivity requirements on BXs.

1. The PutGeT law (cf. Proposition 1) implies that get is surjective on the view type $V$ and "put $s$ " is injective for all sources $s$. If the PUTGET law holds then, for all sources $s$, get is a left inverse of "put s" and "put s" is a right inverse of get.

2. The StrongGetPut law (cf. Proposition 1) implies that get is injective and "put $s$ " is surjective on the source type $S$ for all sources $s$. If the STRONGGETPUT law holds, then, for all sources $s$, get is a right inverse of "put s" and "put s" is a left inverse of get.

Definition 7 (Bijectivity and inverse). A function $f$ is bijective if and only if it is injective and surjective. In this case, there is exactly one left inverse $g$ of $f$. It is also a right inverse of $f$ and, therefore, called inverse, for short. We write $f^{-1}$ for the inverse of a bijective function $f$.

Intuitively, a bijective function defines a one-to-one correspondence between its argument and result type. As a consequence, both have the same cardinality.

Proposition 2 justifies why BXs that satisfy both the PUTGET and the STRONGGETPUT law are called bijective, because in this case get is bijective and put $s$ is its inverse for all sources $s$. This shows that put functions in bijective BXs can ignore their source argument, such that they trivially satisfy the PUTPUT law (cf. Definition 3). Therefore, every bijective BX is also very well behaved.

It is worth considering the differences between the properties defined in Definitions 577 for multiargument functions in curried and uncurried style. For example, for a given put function, we can distinguish the following notions of injectivity: 
1. put is injective

2. put $s$ is injective for all source values $s$

3. uncurry put is injective

We can build an intuition for the differences between these properties by considering the cardinalities of source and view types, say $|S|$ and $|V|$, respectively.

The cardinality of the argument type $S$ of put is $|S|$ and the cardinality of its result type $V \rightarrow S$ is $|S|^{|V|}$. Therefore, it is easy to give an injective put function. In fact, the constant put function defined as follows is an example:

$$
\text { put } s v=s
$$

On the other hand, the argument type $(S, V)$ of uncurry put has cardinality $|S| \cdot|V|$ and its result type $S$ has cardinality $|S|$. So, for finite source and view types with $|V|>1$, there is no put function such that uncurry put is injective.

Property 2 lies in-between properties 1 and 3. For source types that are "at least as big as" the view type, there are put functions that satisfy property 2 . The constant put function, however, is a counterexample because all views are mapped to the same source.

We can make similar observations for surjectivity instead of injectivity of multi-argument functions.

Proposition 3 (Surjectivity of uncurry put). The GETPUT law (cf. Definition 1) implies that "uncurry put" is surjective on the source type. Proof. If the GETPUT law holds, then the function " $p^{r}=\lambda s \rightarrow(s$, get $s)$ " is a right inverse of "uncurry put":

$$
\begin{aligned}
& \text { uncurry put }\left(p^{r} s\right) \\
= & \left\{\text { definition of } p^{r}\right\} \\
& \text { uncurry put }(s, \text { get } s) \\
= & \{\text { definition of uncurry }\} \\
= & \text { put } s \text { (get } s) \\
& \{\text { GETPUT law }\}
\end{aligned}
$$

Our final mathematical concept that turns out helpful for characterizing the different classes of BXs introduced in Section 2 is idempotence.

Definition 8 (Idempotence). A function $f:: A \rightarrow A$ is called idempotent if $f \cdot f=f$ holds.

Proposition 4 (Idempotence of ('put' $v$ )). In a well-behaved BX, that is, which satisfies the GETPUT and PUTGET laws, the function ('put' $v$ ) is idempotent for all views $v$. This idempotence requirement can also be expressed as the following equation, called the PUTTwICE law [9]:

$$
\text { put (put s v) } v=\text { put } s v
$$

PutTwice

Proof. The following equations use the PUTGET and GETPUT laws to derive the required idempotence:

$$
\begin{aligned}
& \left(\left({ }^{\prime} p u t^{6} v\right) \cdot\left({ }^{\prime} p u t^{6} v\right)\right) s \\
& =\{\text { function composition }\} \\
& \left(s^{\prime} p u t^{6} v\right) \text { 'put'v } \\
& =\{\text { prefix notation }\} \\
& \text { put (put s v) v } \\
& =\{\text { PutGet law }\} \\
& \text { put (put s v) (get (put s v)) } \\
& =\{\text { GetPUT law } \\
& \text { put } s v \\
& =\{\text { infix operator section }\} \\
& \left({ }^{\prime} p u t^{6} v\right) s
\end{aligned}
$$


Fischer S, et al. Sci China Inf Sci $\quad$ xxxxxx 2014 Vol. 57 xxxxxx:10

The PUTPUT law is stronger than idempotence of ('put' $v$ ) because it requires a similar equation for compositions of put applied to different views $v$ and $v^{\prime}$ instead of compositions of put applied to the same view. Example 2 shows that the PUTPUT law is stronger than idempotence of (' $p u t^{\prime} v$ ) because the BX defined there is well-behaved, but not very.

\section{Characterizing BXs in terms of the putback function}

We now characterize different classes of BXs introduced in Section 2 based on necessary conditions observed in Section 3. From this characterization, we will be able to identify exactly, for each introduced class of BXs, which part of the definition of a BX is redundant due to the corresponding laws.

As a preliminary observation, note that for bijective BXs the put function is uniquely determined by the get function and vice versa. We can define

$$
\text { put } s=\text { get }^{-1}
$$

for all sources $s$ to derive a unique put from get. We can also define

$$
\text { get } s=(\text { put } s)^{-1} s
$$

to derive a unique get from put.

For (very) well-behaved BXs, the get function does not give rise to a unique put function. Examples 1 and 3 are two different very well-behaved BXs with the same put function. Hence, (very) well-behaved BXs cannot be specified completely by only providing a get function.

In the remainder of this section, we show that (very) well-behaved BXs can be specified completely by giving their put function. No additional conditions on put functions are required apart from necessary conditions observed in Section 3 ,

\subsection{Characterizing well-behaved BXs}

Our first theorem shows that we can replace each of the defining laws for well-behaved BXs by necessary conditions observed previously.

Theorem 1 (Characterizing well-behaved BXs). The following propositions are equivalent:

1. The GETPUT and PUTGET laws hold.

2. The GETPUT law holds, (" $\left.p u t^{\prime} v\right)$ is idempotent for all views $v$, and "put $s$ " is injective for all sources $s$.

3. The PUTGET law holds, ("put'v) is idempotent for all views $v$, and "uncurry put" is surjective on the source type.

Proof. We show the implications $1 \Rightarrow 2 \Rightarrow 1 \Rightarrow 3 \Rightarrow 1$.

$1 \Rightarrow 2$ The idempotence requirement follows from Proposition 4. The injectivity requirement is a direct consequence of the PUTGET law (cf. Proposition 2).

$2 \Rightarrow 1$ To conclude the PUTGET law

$$
\text { get }(\text { put } s v)=v
$$

for all sources $s$ and views $v$, let $p^{l}$ be a left inverse of put (put $s v$ ). Then, verify the following equations using the GETPUT law and Proposition 4

$$
\begin{aligned}
& \operatorname{get}(\text { put } s v) \\
& \{\text { definition of } i d\} \\
& i d(\text { get }(\text { put } s v))
\end{aligned}
$$


Fischer S, et al. Sci China Inf Sci $\quad \operatorname{xxxxxx} 2014$ Vol. $57 \operatorname{xxxxxx:11}$

$$
\begin{aligned}
= & \left\{p^{l} \text { is left inverse of put }(\text { put } s v)\right\} \\
= & p^{l}(\text { put }(\text { put } s v)(\text { get }(\text { put } s v))) \\
& p^{l}(\text { Gut } s v) \\
= & \left\{\text { idempotence of }\left({ }^{l} p u t^{\prime} v\right), \text { Proposition } 4\right\} \\
= & p^{l}(\text { put }(\text { put } s v) v) \\
& \left.i d p^{l} \text { is left inverse of put }(\text { put } s v)\right\} \\
= & \{\text { definition of } i d\}
\end{aligned}
$$

$1 \Rightarrow 3$ The idempotence requirement follows from Proposition 4 Surjectivity of "uncurry put" follows from Proposition 3

$3 \Rightarrow 1$ To conclude the GETPUT law, let $p^{r}$ be a right inverse of "uncurry put", and for a source $s$ define " $\left(s^{\prime}, v\right)=p^{r} s$ " such that "put $s^{\prime} v=s$ ". Then, verify the following equations using the PUTGET law and Proposition 4

$$
\begin{aligned}
& \text { put } s(\text { get } s) \\
= & \left\{\text { put } s^{\prime} v=s\right\} \\
& \text { put }\left(\text { put } s^{\prime} v\right)\left(\text { get }\left(\text { put } s^{\prime} v\right)\right) \\
= & \{\text { PUTGET law }\} \\
& \text { put }\left(\text { put } s^{\prime} v\right) v \\
= & \left\{\text { idempotence of }\left({ }^{\prime} p u t^{\prime} v\right), \text { Proposition } 4\right\} \\
& \text { put } s^{\prime} v \\
= & \left\{\text { put } s^{\prime} v=s\right\} \\
& s
\end{aligned}
$$

We cannot replace both defining laws for well-behaved BXs by the necessary conditions on the put function without losing the connection to the get function. However, those necessary conditions on put functions are sufficient in the sense that they give rise to a unique get function such that the resulting BX is well-behaved. The following Theorem 2 shows that put functions satisfying the necessary conditions used in Theorem 1 characterize well-behaved BXs, that is, well-behaved BXs are uniquely determined by their putback function.

Theorem 2 (Uniqueness of get for well-behaved $p u t$ ). Assume a put function that satisfies all of the following propositions:

1. ('put' $v$ ) is idempotent for all views $v$.

2. "put $s$ " is injective for all sources $s$.

3. "uncurry put" is surjective on the source type.

Then, the following propositions are also satisfied:

(a) For every source $s$, there is exactly one view $v$ such that put $s v=s$.

(b) There is exactly one get function such that the resulting BX is well-behaved.

Proof.

(a) Regarding the existence of $v$, choose for all $s$ a source $s^{\prime}$ and a view $v$ such that $s=p u t s^{\prime} v$ according to (3). Then, the following equations hold because of Proposition 4

$$
\begin{aligned}
& \quad p u t s v \\
& =\left\{s=\text { put } s^{\prime} v\right\}
\end{aligned}
$$


Fischer S, et al. Sci China Inf Sci $\quad \operatorname{xxxxxx} 2014$ Vol. $57 \operatorname{xxxxxx:12}$

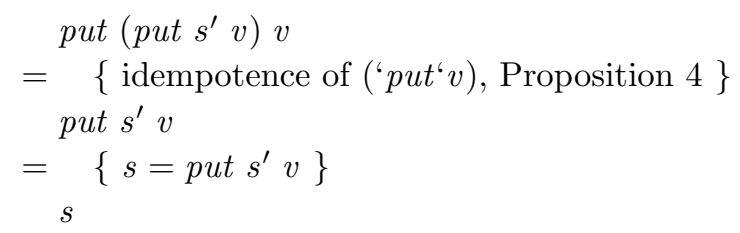

A view $v$ satisfying the equation put $s v=s$ is unique because "put $s$ " is injective according to (2).

(b) Regarding the existence of get define

get $s=v$, with $v$ such that $s=$ put $s v$

according to $(a)$. Then, we can verify the GETPUT law as follows:

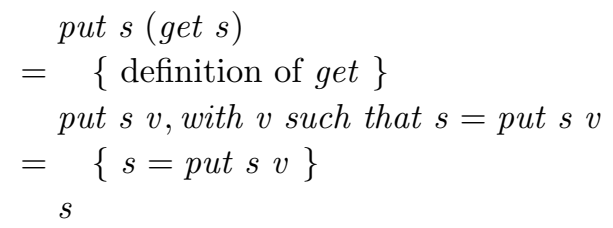

From the second proposition of Theorem 1, we can now conclude that the resulting BX is wellbehaved.

Regarding the uniqueness of get, consider get $^{\prime}$ such that the resulting BX is well-behaved. Then, we can observe the following equations making use of $(a)$ and the PUTGET law:

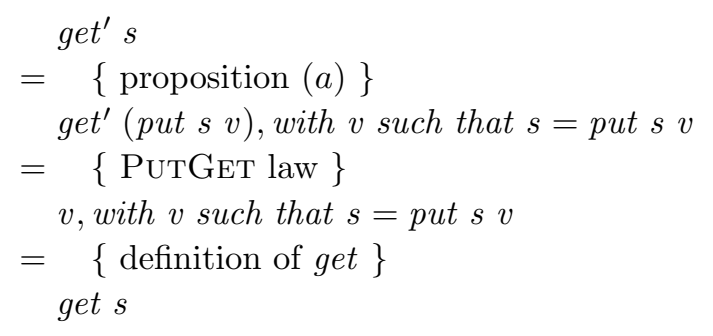

This result shows that well-behaved BXs are characterized by their putback function, that is, the get function is redundant for the purpose of specification.

\subsection{Characterizing very well-behaved $\mathrm{BXs}$}

Very well-behaved BXs differ from well-behaved BXs only in the PUTPUT law (see Definition 3) which (as we have observed after Proposition 44) is a stronger version of the idempotence requirement on (' $p u t^{6} v$ ) for all views $v$. That said, the following characterization of very well-behaved BXs is a direct consequence of Theorem 1 .

Corollary 1 (Characterizing very well-behaved BXs). The following propositions are equivalent:

1. The GetPut, PutGet, and PutPut laws hold.

2. The GETPUT and PUTPUT laws hold and "put $s$ " is injective for all sources $s$.

3. The PutGeT and PutPuT laws hold and "uncurry put" is surjective on the source type.

Proof. Direct consequence of Theorem1 1 because the PUTPUT law (cf. Proposition[1) implies idempotence of ('put' $v$ ) for all views $v$.

We get a similar result regarding the uniqueness of the get function for a given put function of a very well-behaved BX as we observed for well-behaved BXs. 
Corollary 2 (Uniqueness of get for very well-behaved put). Assume a put function that satisfies all of the following propositions:

1. The PUTPUT law holds.

2. "put $s$ " is injective for all sources $s$.

3. "uncurry put" is surjective on the source type.

Then, there is exactly one get function such that the resulting BX is very well-behaved.

Proof. The propositions imply those of Theorem 2 so there is a unique get function such that the resulting BX is well-behaved. It is also very well-behaved because the put function satisfies PUTPUT according to proposition (1).

\subsection{Partial BXs}

So far, we have assumed get and put to be functions - in the mathematical sense - between a source type $S$ and a view type $V$. This entails, specifically, that get and put are both total, that is, yield a value for every argument in their respective domains. In the case of well-behaved BXs, totality of get means surjectivity of uncurry put and totality of put means surjectivity of get.

While it is possible, in principle, to define precise types that match the domain and range of arbitrary get and put functions exactly, in practice it is often convenient to allow the source and view types of BXs be larger and define get or put as partial functions between these larger types [26].

Example 4 (Partial BX to access the head of a non-empty list). As an example for a partial BX, consider the following definitions:

$$
\begin{aligned}
& \text { getHead }\left(x:-{ }_{-}\right)=x \\
& \text { putHead }(-: x s) x=x: x s
\end{aligned}
$$

In Haskell, $(x: x s)$ denotes a list containing at least one element $-x$ being the first and $x s$ containing all remaining ones.

Here are some example calls that demonstrate the behaviors of the forward and backward functions using an alternative list notation.

$$
\begin{aligned}
& \text { getHead }[1,2,3]=1 \\
& \text { getHead [] -- fails } \\
& \text { putHead }[1,2] 3=[3,2] \\
& \text { putHead [] } 1 \text {-- fails }
\end{aligned}
$$

If we define the source type for this transformation to be the type [Elem] of lists of elements of type Elem and the view type to be Elem, then getHead and putHead do not form a well-behaved BX. The GETPUT and PUTGET laws are violated if we use the empty list as a source value.

$$
\begin{aligned}
& \text { putHead }[](\text { getHead }[]) \neq[] \\
& \text { getHead }(\text { putHead }[] 1) \neq 1
\end{aligned}
$$

Also, note that one of the conditions on the put function used in Theorem 2 is violated: "uncurry putHead" is not surjective because put $s v \neq[]$ for all $s$ and $v$.

In Example 4, however, there is an easy way out. If we define the source type to be the type of non-empty lists, then putHead does satisfy all conditions of Theorem 2 and getHead is the unique forward function forming a well-behaved BX with putHead.

In general, we may assume such precise types even if we do not express them in a programming language. For example, in Section 5 we will define a BX whose view type consists of people from Tokyo. While we do not model the type of people from Tokyo in our programming language of choice, we can still use 
Fischer S, et al. Sci China Inf Sci $\quad \operatorname{xxxxxx} 2014$ Vol. $57 \operatorname{xxxxxx:14}$

Theorem 2 to derive a corresponding forward function automatically via the same reasoning we applied to Example 4

Alternatively, we could reformulate our definitions such that get and put are partial functions and bidirectional laws are partial equalities. This relaxation still preserves all the above theorems (excluding surjectivity of get and of uncurry put) as long as get and put are safe [26, in the sense that get is defined for the image of put and put is defined for the image of get. For space and readability reasons, we refrain from restating our results with partial functions.

\section{$5 \quad$ Put-based programming of BXs}

Our main result, Theorem 2 states that in a well-behaved BX the definition of get is redundant given a definition of put that satisfies certain properties necessary for well-behavedness. In this section, we argue for specifying a well-behaved BX by defining a put function. We show how to use the functional-logic programming language Curry [12] to derive a corresponding get function automatically and show, using several examples, how to define put functions for well-behaved BXs.

\subsection{Using Curry to derive get from put}

We can use the built-in search facilities of Curry to derive the get function of a well-behaved BX from their put function automatically. While search is probably not the most efficient way to obtain get from put, it is sufficient for the demonstration purposes of this section.

Syntactically, Curry is an extension of basic Haskell. Semantically, an important difference is the handling of pattern matching - especially in the presence of multiple rules. In Curry, pattern-match failure is handled silently and more than one defining rule of a function (or more accurately: operation) may be applied nondeterministically. For example, the following program splits a given list into two parts at an arbitrary position:

$$
\begin{array}{ll}
\text { split } x s & =([], x s) \\
\text { split }(x: x s) & =(x: y s, z s) \\
\text { where } & \\
\quad(y s, z s) & =\text { split } x s
\end{array}
$$

The defining rules of split are overlapping but, unlike in Haskell, not only the first matching rule is applied but all matching rules are applied nondeterministically. For example, there are three possible results of the call split $[1,2]$ :

$$
\begin{aligned}
& ([1,2],[]) \\
& ([1],[2]) \\
& ([],[1,2])
\end{aligned}
$$

Implementations of Curry usually allow to observe all nondeterministic results interactively and are free to present them in an arbitrary order.

An alternative way to define split is by constraining free variables. Instead of encoding nondeterminism using overlapping rules, we can also induce search by calculating with unknown information. Here is an alternative definition of split following this approach.

$$
\begin{aligned}
& \text { split } x s \mid x s==y s+z s=(y s, z s) \\
& \text { where } \\
& y s, z s \text { free }
\end{aligned}
$$

This definition expresses in a guard that concatenating two unknown lists ys and $z s$ using the predefined operation "\#" should yield the argument list xs. The Curry implementation searches for instantiations

2) Because of nondeterminism, the input-output relation of a Curry operation does not necessarily describe a function. 
of $y s$ and $z s$ that satisfy the guard and returns them as result of split. As there are, generally, multiple ways to instantiate $y s$ and $z s$ to satisfy the guard, the result of split is nondeterministic like with previous definition 3

To define the get function of a BX based on a put function, we can use the same programming style as in the second definition of split. For convenience, we first define a type for BXs between a source type $s$ and a view type $v$.

type $B X s v=s \rightarrow v \rightarrow s$

The type $B X s v$ defines BXs as their put function. We provide a function put that just calls this function.

$$
\begin{aligned}
& \text { put }:: B X s v \rightarrow s \rightarrow v \rightarrow s \\
& \text { put bx } s v=b x s v
\end{aligned}
$$

The function get is defined based on put using the constraint given in Theorem 2 .

$$
\begin{aligned}
& \text { get }:: B X s v \rightarrow s \rightarrow v \\
& \text { get } b x s \mid \text { put bx s } v==s=v \\
& \text { where } \\
& \quad v \text { free }
\end{aligned}
$$

These definitions allow to define BXs by giving only the putback function but to use them in both directions. In the remainder of this section, we show several examples of BXs in this putback style.

\subsection{Record field access}

The most basic BXs access fields of records similarly to Example 1. To demonstrate record field access, we define a type for people.

$$
\begin{aligned}
& \text { data Person }=\text { Person Name City } \\
& \begin{aligned}
\text { data Name } & =\text { Hugo } \mid \text { Sebastian } \mid \text { Zhenjiang } \\
\text { data City } & =\text { Braga } \mid \text { Kiel } \mid \text { Tokyo }
\end{aligned}
\end{aligned}
$$

Example 5. We define the BXs name and city that access the name and associated city of a person.

$$
\begin{aligned}
& \text { name }:: \text { BX Person Name } \\
& \text { name }(\text { Person }-c) n=\text { Person } n c \\
& \text { city }:: \text { BX Person City } \\
& \text { city }\left(\text { Person } n_{-}\right) c=\text { Person } n c
\end{aligned}
$$

Both definitions specify a BX by its putback function and we can issue calls in the Curry system KiCS2 to check that the derived forward function works as expected.

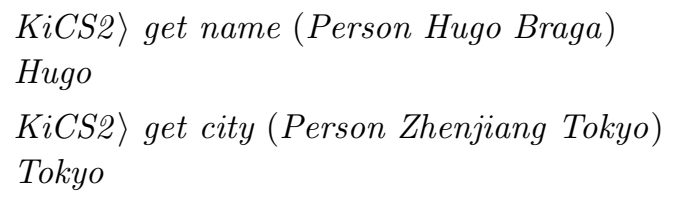

Of course, we can also call the putback function — directly or indirectly using put - as the following examples demonstrate.

KiCS2〉 put city (Person Sebastian Tokyo) Kiel

Person Sebastian Kiel

3) Depending on the Curry implementation, constraint equalities need to be specified using a different operator. We use the Kiel Curry System KiCS2 20] which allows to apply standard equality to free variables. 
KiCS2〉 city (Person Sebastian Tokyo) Kiel

Person Sebastian Kiel

We can also use these BXs in other Curry functions. For example, the following predicate checks whether a person is from a given city:

isFrom $::$ City $\rightarrow$ Person $\rightarrow$ Bool

isFrom $c p=c=$ get city $p$

In the following, we define BXs in analogy to database view updates using a database of people.

\subsection{Database view updating}

Historically, database view updating is a primary source of motivation for researching BXs. We now demonstrate how different view update strategies described in the literature can be expressed in our put-based framework for bidirectional programming.

For the sake of simplicity, we regard database tables as sets of rows represented as lists sorted by a key identifying rows uniquely. For example, the following is a database of people where each person is identified by their name:

$$
\begin{aligned}
& \text { people }=[\text { hugo, sebastian, zhenjiang }] \\
& \text { hugo }=\text { Person Hugo Braga } \\
& \text { sebastian }=\text { Person Sebastian Tokyo } \\
& \text { zhenjiang }=\text { Person Zhenjiang Tokyo }
\end{aligned}
$$

To update this database of people, the following function mergePeople will be helpful. It takes two tables of people (sorted by name) and merges them into a single (sorted) table of people. If entries with the same key are present in both tables, then the entry in the second table overwrites the entry in the first.

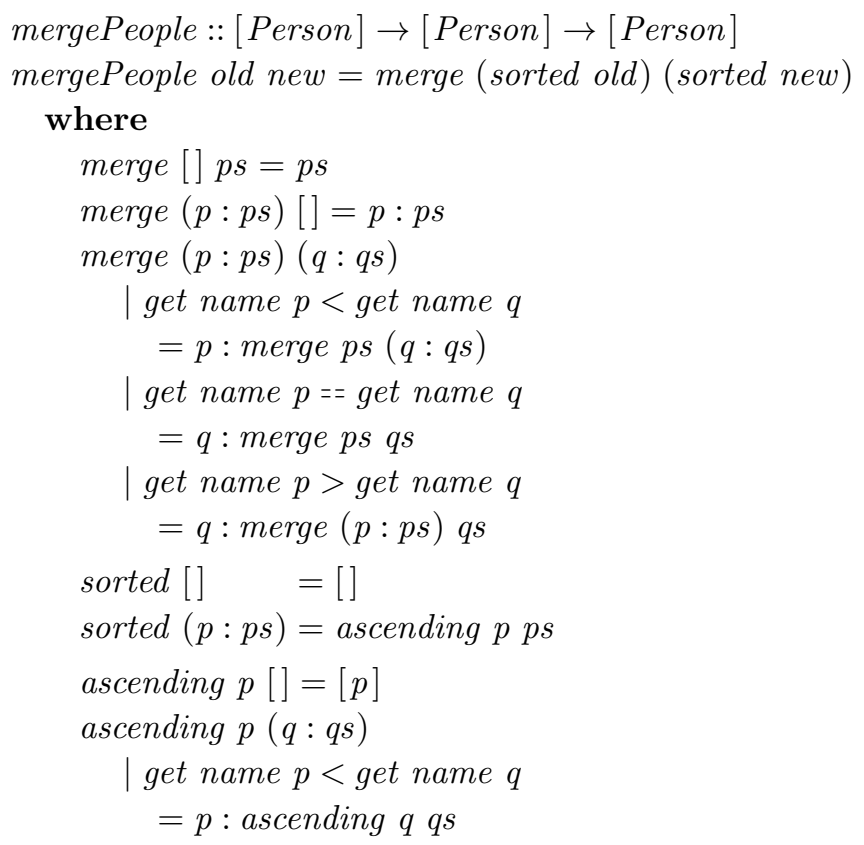

The function mergePeople restricts the tables passed as arguments to be sorted using the partial function sorted that ensures that its argument is a sorted list of people. The function sorted is the identity function on sorted tables but fails on lists of people that are not sorted by name (in mathematical terms, a coreflexive relation that is a subset of the identity relation). 
Fischer S, et al. Sci China Inf Sci $\quad \operatorname{xxxxxx} 2014$ Vol. $57 \operatorname{xxxxxx:17}$

Now, consider a database query that selects all people from a certain city. For example, selecting all people from Tokyo from the people database defined above would result in the following view of this database:

\section{[Person Sebastian Tokyo, Person Zhenjiang Tokyo]}

When adding new people to this view, reflecting it in the original database is straightforward: if a person with the same name already exists, then change their city to Tokyo; if there is no person with that name in the original database, then add it ${ }^{4}$. For deletion, however, there is no straightforward update strategy. When deleting a person from the view, we can either

1. delete it from the original database or

2. change their city to a city different from Tokyo.

Keller [19] argues that both strategies may be reasonable depending on context, so a system computing an update strategy automatically solely based on the definition of the view function is insufficient. Using put-based bidirectional programming, both strategies above can be expressed in a straightforward way.

\subsubsection{Reflecting deletions via deletions}

The first strategy — deleting people from the original database that are not present in the updated viewcan be implemented as follows:

- first, delete all people from the given city from the original database,

- then update the result by merging all people from the updated view.

Example 6 (Reflecting deletions via deletions). The following function peopleFrom implements a BX using the strategy described above 5 )

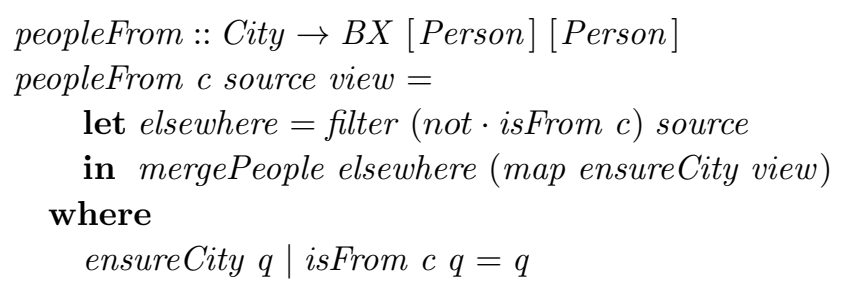

The function peopleFrom uses mergePeople to merge the list elsewhere of people not from the given city in the original source with the list of people from the updated view. The function mergePeople ensures that both lists are sorted. Additionally, peopleFrom restricts updated views using the local function ensureCity, which is a partial identity function on people from the given city. Restricting updated views is important for maintaining the injectivity requirement of Theorem 2 as we discuss below. As a consequence of this restriction, it is not possible to move people to a different city using this update strategy.

Conceptually, peopleFrom $c$ is a BX between the source type of sorted lists of people and the view type of sorted lists of people from the city $c$. Note that specifying the view type in the type system would require a dependently typed programming language, that is, one where the types of expressions can depend on the values of others. Curry is not dependently typed, so we define peopleFrom $c$ as a partial function instead.

To verify that the get-function derived by our framework is unique and the resulting transformation is well-behaved, we need to check the following three conditions:

4) In fact, these are not the only well-behaved ways to handle additions in a view. Changing the city of an existing person could be distinguished from deleting it and adding a new person if people would have additional properties besides their name and city. Also, adding people not from Tokyo would not violate well-behavedness if only performed when a person in the view is not present in the source. While our approach allows to define all such updates, we restrict ourselves to more reasonable strategies trying to keep updates minimal, which is consistent with update translation strategies used for database view updating.

5) The predefined function map applies a given function to each element of a list. 
1. peopleFrom $c$ (peopleFrom $c s v$ ) $v=$ peopleFrom $c s v$ for all cities $c$, sorted lists $s$ of people, and sorted lists $v$ of people from the city $c$,

2. peopleFrom $c s$ is injective for all cities $c$ and sorted lists of people $s$, and

3. uncurry (peopleFrom $c$ ) is surjective on sorted lists of people for all cities $c$.

The first condition is satisfied because the second application of peopleFrom $c$ will remove the updated people and then readd them, so updating twice using the same view is the same as updating only once.

The second condition is satisfied because peopleFrom $c$ restricts updated views to people from the given city and is not defined for other views. Without this restriction, views that contain people from the original database not from the city $c$ would map to the same updated source as views not containing them, violating the injectivity requirement. By deleting all people from the city $c$ from the original source and ensuring that people in the updated view are from the city $c$, peopleFrom $c$ ensures that updated views are mapped uniquely to updated sources.

The third requirement is satisfied because every database of people can be obtained using peopleFrom $c$ by passing it as original source together with a view that includes all its people from the city $c$ 6)

Together, these conditions ensure that the get function derived for peopleFrom $c$ is unique and the resulting BX is well-behaved. The following calls demonstrate the behavior of the derived view function and the defined update strategy:

KiCS2〉 get (peopleFrom Tokyo) people

[Person Sebastian Tokyo, Person Zhenjiang Tokyo]

KiCS2〉 put (peopleFrom Tokyo) people [zhenjiang]

[Person Hugo Braga, Person Zhenjiang Tokyo]

KiCS2〉 put (peopleFrom Tokyo) people [put city hugo Tokyo, zhenjiang]

[Person Hugo Tokyo, Person Zhenjiang Tokyo]

The first call demonstrates the get function querying all people from Tokyo in the database defined earlier. The second call demonstrates deleting sebastian by deleting him from the updated view passed to the put function. The result contains hugo who is not from Tokyo and zhenjiang who was included in the updated view. The third call demonstrates deleting sebastian and moving hugo to Tokyo by adding him to the list of people from Tokyo.

\subsubsection{Reflecting deletions via modifications}

Instead of deleting people from the database that are not present in the updated view queried by city, we can also move them to a different city. This strategy can be implemented as follows:

1. first move all people from the queried city to the new city in the original source,

2. then call the update strategy defined in Section 5.3.1 to overwrite all moved people who are present in the view, effectively moving only those who are not present.

Example 7 (Reflecting deletions via modifications). The following definition implements the strategy described above:

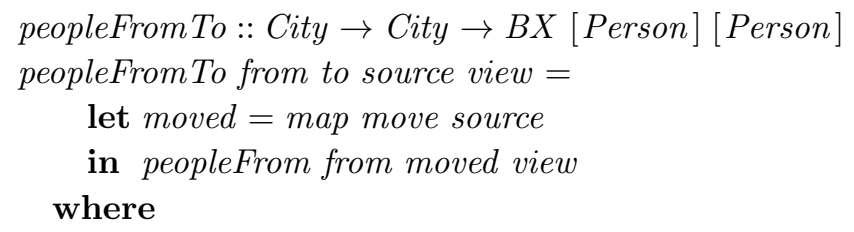

6) This requirement would not be necessary in a reformulation of Theorem 2 for partial functions, but it ensures that the domain of the forward function (i.e., the range of the uncurried backward function) is the "type" of all sorted lists of people, as intended. 


$$
\begin{aligned}
& \text { Fischer S, et al. Sci China Inf Sci } \text { xxxxxx } 2014 \text { Vol. } 57 \text { xxxxxx:19 } \\
& \text { move } p \mid \begin{array}{ll}
\text { get city } p==\text { from } & =\text { put city } p \text { to } \\
\mid \text { otherwise } & =p
\end{array}
\end{aligned}
$$

Again, we need to verify the conditions of Theorem 2 to ensure that the resulting BX is well-behaved:

1. Putting an original source with the same view twice is the same as updating it only once because the underlying transformation peopleFrom satisfies this property for all sources, that is, also for the modified source passed by peopleFromTo.

2. The injectivity requirement is also implied by the corresponding property for the underlying transformation.

3. Regarding surjectivity, note that, even though people in the original source are moved initially, they can be moved back by including all moved people in the view argument. So, obtaining an arbitrary source as result of peopleFrom To is achieved in the same way as for peopleFrom.

Here are example calls that demonstrate the difference between the peopleFrom strategy and the peopleFrom To strategy.

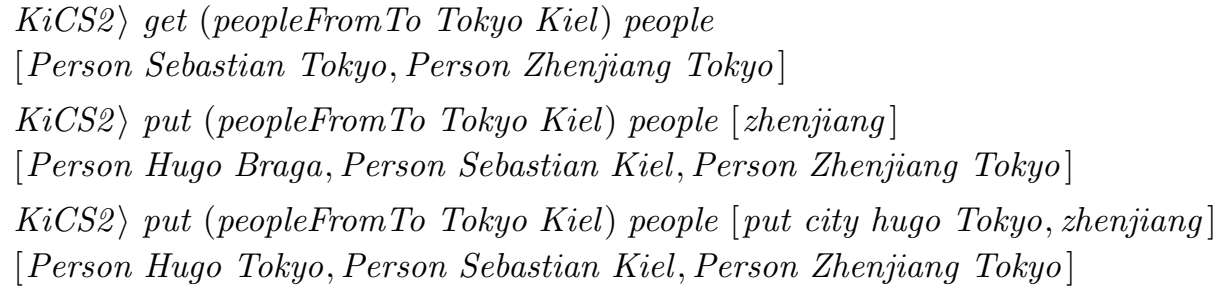

The get function of the BX peopleFromTo Tokyo Kiel is the same as the get function of peopleFrom Tokyo. The difference is only in the put function which moves sebastian from Tokyo to Kiel when he is deleted from the view instead of deleting him from the source. Inserted people, like hugo, are inserted into the original source (or modified if they already exist) just like before.

\section{Related work}

In the last 10 years, various bidirectional programming languages [6, 16] have become increasingly popular across a wide range of communities, including data synchronization, model transformations, graph transformations, relational databases, and functional programming. We review only a few that are more related to our work.

The pioneering work by Foster et al. 10. proposes one of the first bidirectional programming languages for tree-structured data. They recast many of the ideas for database view-updating into the design of a language of BXs named lenses, consisting of a get and a put function that satisfy well-behavedness laws analogous to the ones proposed in [7, 1. The novelty of their work is by putting emphasis on types and totality of lens transformations, and by proposing a series of combinators that allow reasoning about totality and well-behavedness of lenses in a compositional way. Our paper studies precisely total (very) well-behaved lenses.

For relational data built using standard SPJ (selection-projection-join) relational algebra combinators and composition, the relational lenses [4] are proposed to provide one possible update policy based on a careful treatment of functional dependencies, together with a type system using record predicates and functional dependencies to express the exact conditions on the source and view schemas under which lenses are total and well-behaved.

Boomerang [3] is a language for the BX of string data, built using a set of regular operations and a type system of regular expressions. To overcome issues with order, their lens combinators adopt an update translation strategy based on keys that are introduced by the programmer in the form of annotations to lens expressions. Matching lenses [2] generalize the string lens language by lifting the update translation 
Fischer S, et al. Sci China Inf Sci $\quad \operatorname{xxxxxx} 2014$ Vol. $57 \operatorname{xxxxxx:20}$

strategy from a key-based matching to support a set of different alignment heuristics that can be chosen by users.

Pacheco and Cunha 27. propose a point-free functional language of total well-behaved lenses, using a simple positional update strategy, and later Pacheco et al. 28, generalize the matching lenses approach to infer and propagate insertion and deletion updates over such language.

Hidaka et al. 13] propose the first linguistic approach for bidirectional graph transformations by giving a bidirectional semantics to the UnCal graph algebra. Although their base semantics is compositional, they process deletions in the view by locating the correlated subgraph in the source, and for insertions in the view they have a dialog with the user at view update time to calculate the correlated inserted subgraph in the source.

All existing bidirectional programming approaches based on lenses focus on writing bidirectional programs that resemble writing the get function, and possibly take some additional parameters that provide limited control over the update strategy of the put function. Since these languages are state-based, the put function of a lens must align the updated view and the original source structures to identify the modifications and translate them to the source accordingly. Although for unordered data (relations, graphs) such alignment can be done rather straightforwardly, for ordered data (strings, trees) it is more problematic to find a reasonable alignment strategy, and thus to provide a reasonable view update translation strategy. Our results open the way to put programming languages, that in theory could give the programmer the possibility to express all well-behaved update translation strategies.

In his $\mathrm{PhD}$ thesis, Foster [9] independently discusses a characterization of lenses in terms of put functions (considering only total get and put functions), in point-wise terms, similar to our main theorem. Interestingly, he arrives at a notion of put semi-injectivity that is slightly stronger than our injectivity of put s. He also uses the PUTTWICE law which we identify as idempotence of ' $p u t^{\text {' }} v$. Nevertheless, he does so only to plead for a forward programming style and does not pursue a putback programming style. Also, he advocates that both styles are equivalent because writing a bidirectional program in a get-based bidirectional language is the same as writing a backward transformation. We disagree on the grounds that they are pragmatically distinct, as programmers of a BX in an existing get-based bidirectional language are limited by the language designer in their knowledge and control of the backward update strategy, and thus are supplied with "a" backward transformation and not "the" backward transformation. Our emphasis on putback programming highlights that programmers must be both aware of and responsible for the backward update strategy to specify a BX completely. We explore the putback style to demonstrate this difference and illustrate a possible way to derive get functions from put functions.

This paper is a revised version of the technical report [8, which has inspired some work on designing domain-specific languages for supporting putback style bidirectional programming [29, 30].

\section{Conclusions and future work}

In this paper, we characterize the class of (very) well-behaved BXs solely based on their putback functions. In doing so, we rephrase existing laws for BXs based on simple mathematical concepts such as injectivity, surjectivity, and idempotence. We use our characterization to show that (very) well-behaved BXs are uniquely determined by their backward functions and corresponding forward functions can be obtained automatically. In sharp contrast to bidirectional programming approaches based on get, writing put is sufficient to express all (very) well-behaved BXs for a given get, and it is indeed more powerful than writing get alone because of its full control over the bidirectional behavior. Although combinatorial bidirectional programming approaches can theoretically achieve the same expressiveness [29], by providing a seemingly unbounded choice of different behaviors via different combinators, they only provide a more indirect and less intuitive way of writing a desired put function.

Following this putback-based characterization of BXs, we show how to directly realize existing update strategies as putback functions. We use the built-in search facilities of the functional-logic programming language Curry to obtain the get function corresponding to a user-defined put function that satisfies necessary conditions for well-behavedness. We informally argue that our definitions satisfy these well- 
Fischer S, et al. Sci China Inf Sci $\quad \operatorname{xxxxxx} 2014$ Vol. $57 \operatorname{xxxxxx:21}$

behavedness properties and discuss, based on a more complicated example, that ensuring them can be difficult without further assistance.

It is worth noting that writing put should be more difficult than writing get in general, because put essentially contains get (i.e., get can be uniquely determined by put). However, our recent work [30] shows that it is possible to simplify development of put based on existing update languages.

For our future work, the first immediate direction is to investigate the design of a general put programming language that can guide users by only allowing them to define well-behaved BXs but retain the full power of writing put. Preliminary research towards this direction has been reported in 18. Another interesting direction would be to investigate how to extend our theory to consider view side effects. Some bidirectional programming approaches [19, 22, 13, relax the requirement imposed by PUTGET to admit view side effects in some particular cases, instead of disallowing view updates.

\section{Acknowledgments}

This work is supported financially by the Nation Basic Research Program (973 Program) of China (grant No. 2015CB352201) and by JSPS Grant-in-Aid for Scientific Research (A) No. 25240009 in Japan.

\section{References}

1 Bancilhon F, Spyratos N. Update semantics of relational views. ACM Transactions on Database Systems, 1981. 6(4):557-575

2 Barbosa D M J, Cretin J, Foster J N, Greenberg M, Pierce B C. Matching lenses: alignment and view update. In: Proceedings of ICFP 2010, Baltimore, Maryland, USA, 2010. 193-204

3 Bohannon A, Foster J N, Pierce B C, Pilkiewicz A, Schmitt A. Boomerang: resourceful lenses for string data. In: Proceedings of POPL 2008, San Francisco, USA, 2008. 407-419

4 Bohannon A, Pierce B C, Vaughan J A. Relational lenses: a language for updatable views. In: Proceedings of PODS 2006, Chicago, Illinois, USA, 2006. 338-347

5 Buneman P, Cheney J, Vansummeren S. On the expressiveness of implicit provenance in query and update languages. ACM Transactions on Database Systems, 2008. 33(4):28:1-28:47

6 Czarnecki K, Foster J N,Hu Z, Lämmel R, Schürr A, Terwilliger J. Bidirectional transformations: A cross-discipline perspective. In Proceedings of ICMT 2009, ETH Zurich, Switzerland, 2009. LNCS 5563:260-283

7 Dayal U, Bernstein P. On the correct translation of update operations on relational views. ACM Transactions on Database Systems, 1982. 7:381-416

8 Fischer S, Hu Z, and Pacheco H. "Putback" is the Essence of Bidirectional Programming. GRACE Technical Report 2012-08, National Institute of Informatics, 2012

9 Foster J N. Bidirectional programming languages. PhD thesis, University of Pennsylvania, 2009

10 Foster J N, Greenwald M B, Moore J T, Pierce B C, Schmitt A. Combinators for bidirectional tree transformations: A linguistic approach to the view-update problem. ACM Transactions on Programming Languages and Systems, 2007. 29(3):article 17

11 Gottlob G, Paolini P, Zicari R. Properties and update semantics of consistent views. ACM Transactions on Database Systems, 1988. 13(4):486-524

12 Hanus M. Curry: An integrated functional logic language (vers. 0.8.3), Technical Report, Keil University 2012. Available at: http://www.informatik.uni-kiel.de/ curry/papers/report.pdf 
Fischer S, et al. Sci China Inf Sci $\quad$ xxxxxx 2014 Vol. 57 xxxxxx:22

13 Hidaka S, Hu Z, Inaba K, Kato H, Matsuda K, Nakano K. Bidirectionalizing graph transformations. In: Proceedings of ICFP 2010, Baltimore, Maryland, USA, 2010. 205-216

14 Hofmann M, Pierce B C, and Wagner D. Symmetric lenses. In: Proceedings of POPL 2011, Austin, USA, 2011. 371-384

15 Hofmann M, Pierce B C, Wagner D. Edit lenses. In: Proceedings of POPL 2012, Philadelphia, USA, 2012. $495-508$

16 Hu Z, Schürr A, Stevens P, Terwilliger J F. Dagstuhl seminar on bidirectional transformations (BX). SIGMOD Record, 2011. 40(1):35-39

$17 \mathrm{Hu} \mathrm{Z}$, Mu S C, Takeichi M. A programmable editor for developing structured documents based on bidirectional transformations. Higher-Order and Symbolic Computation, 2008. 21(1-2):89-118

$18 \mathrm{Hu} \mathrm{Z}$, Pacheco H, Fischer S. Validity checking of putback transformations in bidirectional programming. In: Proceedings of FM 2014, Singapore, 2014. LNCS 8442:1-15.

19 Keller A. Choosing a view update translator by dialog at view definition time. In: Proceedings of the 12th International Conference on Very Large Databases (VLDB 86), Kyoto, Japan, 1986. 467-474

20 KiCS2 developers. The kiel curry system, 2012. Available at: http://www-ps .informatik.uni-kiel. de/kics2/.

21 Lämmel R. Coupled software transformations (extended abstract). In: Proceedings of first international workshop on software evolution transformations, Delft, the Netherlands, 2004. 31-35

22 Larson J A, Sheth A P. Updating relational views using knowledge at view definition and view update time. Information Systems, 1991. 16(2):145 - 168

23 Simon Marlow (editor). Haskell 2010 language report. 2010. available at: http://www.haskell.org/ onlinereport/haskell2010/

24 Matsuda K, Hu Z, Nakano K, Hamana M, Takeichi M. Bidirectionalization transformation based on automatic derivation of view complement functions. In: Proceedings of ICFP 200\%, Freiburg, Germany, 2007. 47-58

25 Meertens L. Designing constraint maintainers for user interaction. Manuscript available at http: //www.kestrel.edu/home/people/meertens, 1998.

26 Pacheco H. Bidirectional data transformation by calculation. PhD thesis, University of Minho, 2012.

27 Pacheco H, Cunha A. Generic point-free lenses. In: Proceedings of MPC 2010, Quebec, Canada, LNCS 6120:331-352

28 Pacheco H, Cunha A, Hu Z. Delta lenses over inductive types. Electronic Communications of the EASST, 2012. 49:article 2

29 Pacheco H, Hu Z, Fischer S. Monadic combinators for "putback" style bidirectional programming. In: Proceedings of PEPM 2014, San Diego, California, USA, 2014. 39-50

30 Pacheco H, Zan T, Hu Z. BiFluX: A bidirectional functional update language for XML. In: Proceedings of PPDP '14. Canterbury, UK, 2014.

31 Voigtländer J. Bidirectionalization for free! (pearl). In: Proceedings of POPL 2009, Savannah, Georgia, USA, 2009. 165-176

32 Xiong Y, Liu D, Hu Z, Zhao H, Takeichi M, Mei H. Towards automatic model synchronization from model transformations. In: Proceedings of ASE 200\%, Atlanta, Georgia, USA, 2007. 164-173 